\title{
Efficacy and toxicity in scheduled intravesical gemcitabine versus Bacille Calmette-Guérin for Ta and T1 bladder cancer: a systematic review and meta-analysis
}

\author{
Jun-Lin Lu\#, Qi-Dong Xia", Chen-Qian Liu, Jian-Xuan Sun, Yuan-Yuan Yang, Heng-Long Hu, \\ Shao-Gang Wang
}

Department of Urology, Tongji Hospital, Tongji Medical College, Huazhong University of Science and Technology, Wuhan, China

Contributions: (I) Conception and design: JL Lu, QD Xia; (II) Administrative support: HL Hu, SG Wang; (III) Provision of study materials or patients: JL Lu, QD Xia, HL Hu, SG Wang; (IV) Collection and assembly of data: JL Lu, QD Xia, CQ Liu, JX Sun, YY Yang; (V) Data analysis and interpretation: JL Lu, QD Xia; (VI) Manuscript writing: All authors; (VII) Final approval of manuscript: All authors.

\#These authors contributed equally to this work.

Correspondence to: Heng-Long Hu. Department of Urology, Tongji Hospital, Tongji Medical College, Huazhong University of Science and Technology, Wuhan, China. Email: huhenglong@hust.edu.cn; Shao-Gang Wang. Department of Urology, Tongji Hospital, Tongji Medical College, Huazhong University of Science and Technology, Wuhan, China. Email: sgwangtjm@163.com.

Background: We conducted a meta-analysis to compare the efficacy and toxicity of scheduled intravesical gemcitabine (GEM) and Bacille Calmette-Guérin (BCG) for Ta and T1 non-muscle invasive bladder cancer (NMIBC).

Methods: The database search was performed in Ovid Medline, Embase, Web of Science, Cochrane Library from the commencement of the database to July 7, 2020. Trials using immediate instillation were excluded and we present the included studies in accordance with the PRISMA 2020 reporting checklist. The data extracted was analyzed using Stata 11.0 software.

Results: Six studies of 466 patients comparing GEM and BCG were finally included. No significant difference was detected between GEM and BCG group in recurrence free survival [hazard ratio $(\mathrm{HR})=0.80$, 95\% confidence interval (95\% CI), 0.46-1.37, $\mathrm{P}=0.410$ ], progression free survival (HR =0.82, 95\% CI, 0.381.77, $\mathrm{P}=0.621$ ), and total adverse events [odds ratio $(\mathrm{OR})=0.70,95 \% \mathrm{CI}, 0.38-1.29, \mathrm{P}=0.253)$. However, patients receiving GEM treatment are less likely to develop urinary adverse events, such as dysuria (OR $=0.50,95 \% \mathrm{CI}, 0.29-0.87)$ and hematuria $(\mathrm{OR}=0.40,95 \% \mathrm{CI}, 0.18-0.91)$. We performed subgroup analysis and found that the effects of GEM and BCG were similar even on patients with high recurrence risk tumor. Sensitivity analysis showed the robustness of the results.

Discussion: Scheduled intravesical GEM instillation has a similar effect with BCG instillation in preventing NMIBC recurrence and progression, but GEM therapy causes a lower incidence of dysuria and hematuria than BCG. GEM may be an alternative therapy for BCG. However, the results should be treated with caution due to the low to moderate quality of the included studies.

Keywords: Gemcitabine; Bacille Calmette-Guérin (BCG); non-muscle invasive bladder cancer (NMIBC); metaanalysis; tumor recurrence

Submitted Feb 16, 2021. Accepted for publication Apr 16, 2021.

doi: $10.21037 /$ tcr-21-291

View this article at: https://dx.doi.org/10.21037/tcr-21-291

^ ORCID: Jun-Lin Lu, 0000-0002-3438-0297; Qi-Dong Xia, 0000-0002-2278-1122; Shao-Gang Wang, 0000-0002-3206-1495. 


\section{Introduction}

Intravesical Bacille Calmette-Guérin (BCG) instillation after transurethral resection of bladder tumor (TURBT) has been the standard treatment of intermediate- and high-risk non-muscle invasive bladder cancer (NMIBC) in the last decade (1). However, 35.2-40.4\% of patients will relapse in the short term after BCG instillation (2,3). The patients who progress to muscle invasive bladder cancers (MIBCs), accounting for one-third of NMIBC patients receiving $\mathrm{BCG}$ treatment, have a much poor prognosis and quality of life (3-5). The high incidence of local and systemic complications of BCG instillation negatively impacts patient's compliance and leads to a high proportion of withdrawal $(6,7)$. After two manufacturers announced the halt or severe reduction of BCG production, BCG strain is under a worldwide shortage (8). The development of alternative treatment with better efficacy and tolerance is imperative.

Gemcitabine (GEM) is an antimetabolite pyrimidine analog with a broad spectrum of antitumor activity. Recent researches investigated its preventive effect on tumor recurrence and progression in NMIBC compared with $\mathrm{BCG}$, but indicating opposite outcomes. A randomized controlled trial (RCT) recruited high risk superficial bladder cancer and found that the recurrence rate was $28.1 \%$ for the BCG group and 53.1\% for the GEM group (9). Conversely, another phase 2 RCT revealed that intravesical GEM could significantly improve recurrence-free survival (RFS) than BCG in BCG failure patients with high-risk NMIBC (10). A systematic review with qualitative synthesis is required to clarify whether GEM can be an alternative drug for BCG.

A previous meta-analysis included four RCTs and one case-control study, identifying similar efficacy when compared GEM to BCG (11). The study synthesized evidence using risk ratio instead of hazard ratio (HR), which might ignore time effect for RFS and progressionfree survival (PFS). Furthermore, a multicenter RCT meeting the selection criteria was not included in this study (10). Therefore, the present work aims to evaluate the efficacy and toxicity of scheduled GEM and BCG in patients with NMIBC using extracted HR. We present the following article in accordance with the PRISMA reporting checklist (12) (available at https://dx.doi.org/10.21037/tcr21-291).

\section{Methods}

\section{Data sources and search strategy}

The literature search was conducted by two reviewers (JL Lu and QD Xia). We retrieved four electronic databases (Ovid Medline, Embase, Web of Science, Cochrane Library) from the commencement of the database to July 7, 2020, without language restrictions. Grey literature was searched in the American Society of Clinical Oncology (ASCO) conference abstract, the European Society of Medical Oncology (ESMO) conference abstract, and the National Institute of Health (NIH) website (www.clinicaltrials.gov). The search terms were used in the following strategy: (bladder tumor OR bladder cancer OR urinary bladder neoplasm OR bladder neoplasm OR bladder carcinoma OR urothelium carcinoma OR urothelium tumor) AND (((() non or not) AND (invas* OR invad* OR infiltrat*)) OR noninvas* OR noninvad* OR noninfiltrat*) AND muscle*) OR (cis OR Tis OR Ta OR T1a OR T1b OR superficial)) AND (Bacillus Calmette Guerin OR BCG OR gemcitabine OR GEM). Disagreement during study selection was resolved by consensus with a supervisor (SG Wang).

\section{Inclusion and exclusion criteria}

Both prospective study and retrospective study were eligible. The inclusion criteria were as follows: (I) participants were diagnosed as Ta or $\mathrm{T} 1$ with/without carcinoma in situ NMIBC; (II) intravesical GEM and BCG treatments after TURBT were compared; (III) HRs were available for RFS or PFS. The exclusion criteria were as follows: (I) immediate instillation of GEM; (II) sequential or alternate instillation of intravesical drug; (III) single arm trial. If one research was reported in more than one publication, the most recent study was included.

\section{Data extraction}

Two authors (JL Lu and QD Xia) independently extracted data using a predefined form. The primary outcomes of interest were RFS or PFS after instillation of GEM and BCG. Secondary outcomes were overall survival (OS) or the number of deaths, quality of life, and the number of adverse events. Study characteristics (study design, publication year, country of origin, study period, and the number of patients), 
patient characteristics (sex and age), tumor characteristics (stage, grade, risk of recurrence, history of treatment, and primary/recurrence), intervention (dosage, schedule, and manufacturer), and outcomes were extracted. HRs for RFS and PFS were calculated under the guidance of previous studies $(13,14)$.

\section{Quality assessment}

The quality of RCT was evaluated based on the Cochrane tool for risk of bias assessment (RoB2) (15). The revised RoB2 tool included five domains: the randomization process, deviations from intended interventions, missing outcome data, measurement of the outcome, and selection of the reported result. Non-randomized prospective studies were ranked by the Risk Of Bias In Non-randomized Studies of Interventions (ROBINS-I) (16). The quality of the case-control study was assessed using the NewcastleOttawa Scale criteria (NOS), which includes three main domains: the selection of patients, comparability between groups, and measurement of exposure (17). Disagreements were resolved by discussion with a third author (SG Wang).

\section{Statistical analysis}

The meta-analysis was conducted using Stata 11.0 software. The pooled effect sizes under the random-effect model for RFS and PFS were obtained as HR with $95 \%$ confidence interval (CI) of each study, whereas we used odds ratio (OR) with $95 \%$ CI to estimate the risk of adverse events in patients treated with GEM and BCG. The methodological and clinical heterogeneity were assessed by Cochrane Q statistic and $\mathrm{I}^{2}$ statistic. The heterogeneity was considered significant when $\mathrm{P}<0.05$ and $\mathrm{I}^{2}>50 \%$. Moreover, subgroup analyses were performed in reference to study design, study origin, study center, schedule of therapy, and risk of tumor recurrence. We conducted a sensitivity analysis by omitting the study one by one to assess the robustness of the results.

\section{Results}

\section{Study characteristics}

From 8,639 publications, six studies with 466 patients are finally included (Figure 1) (9,10,18-21). The characteristics of the included studies are summarized in Table 1. Four studies are RCT with moderate to high risk of bias. One study is a non-randomized prospective study of serious risk of bias. One study is a case-control study of high quality. The details of quality assessment can be obtained in supplementary file.

\section{RFS and PFS}

All six-studies report recurrence free survival for GEM and BCG (Figure 2). The efficacy in preventing tumor recurrence is comparable between GEM and BCG [HR, 0.80 (95\% CI, 0.46-1.37), P for effect size, 0.410], showing a high level of heterogeneity (Cochrane $\mathrm{Q}=17.25, \mathrm{P}$ for heterogeneity $\left.=0.004 ; I^{2}=71.0 \%\right)$. Four studies report progression free survival (Figure 2). The pooled effect also demonstrates a comparative efficacy in progression prophylaxis [GEM vs. BCG: HR, 0.82 (95\% CI, 0.38-1.77), $\mathrm{P}$ for effect size, 0.621$]$ with a low level of heterogeneity (Cochrane $\mathrm{Q}=3.50, \mathrm{P}$ for heterogeneity $=0.321 ; \mathrm{I}^{2}=14.3 \%$ ).

\section{Adverse events}

The adverse events during GEM or BCG instillation are dysuria, hematuria, fever, neutropenia-thrombocytopenia, dermatitis, nausea-vomiting, urinary frequency, bladder spasms, urge incontinence, itching, skin rash, cystitis, urosepsis, suprapubic discomfort, and systemic BCG infection. The qualitative synthesis is performed when at least two studies report the same outcome (Figure 3). The meta-analysis shows that the proportion of patients who develop adverse events is comparable between GEM and BCG group [OR, 0.70 (95\% CI, 0.38-1.29), P for effect size, 0.253] with a high level of heterogeneity (Cochrane $\mathrm{Q}=6.50, \mathrm{P}$ for heterogeneity $\left.=0.090 ; \mathrm{I}^{2}=53.8 \%\right)$. However, patients receiving GEM are less likely to develop dysuria [OR, 0.50 (95\% CI, 0.29-0.87), P for effect size, 0.014] or hematuria [OR, 0.40 (95\% CI, 0.18-0.91), P for effect size, 0.028]. Fever events are similar between the two groups [OR, 0.46 (95\% CI, 0.16-1.34), P for effect size, 0.156].

\section{Subgroup analysis and sensitivity analysis}

Subgroup analysis in reference to RFS is conducted to examine the sources of heterogeneity (Table 2). Subgroups include study, intervention, and tumor characteristics, such as study design, study origin, study center, schedule, and risk of recurrence. However, the level of heterogeneity for the subgroup is similar to that for the total effect, indicating that the heterogeneity is not originated from these study features. Figure 4 shows the results of sensitivity analysis 


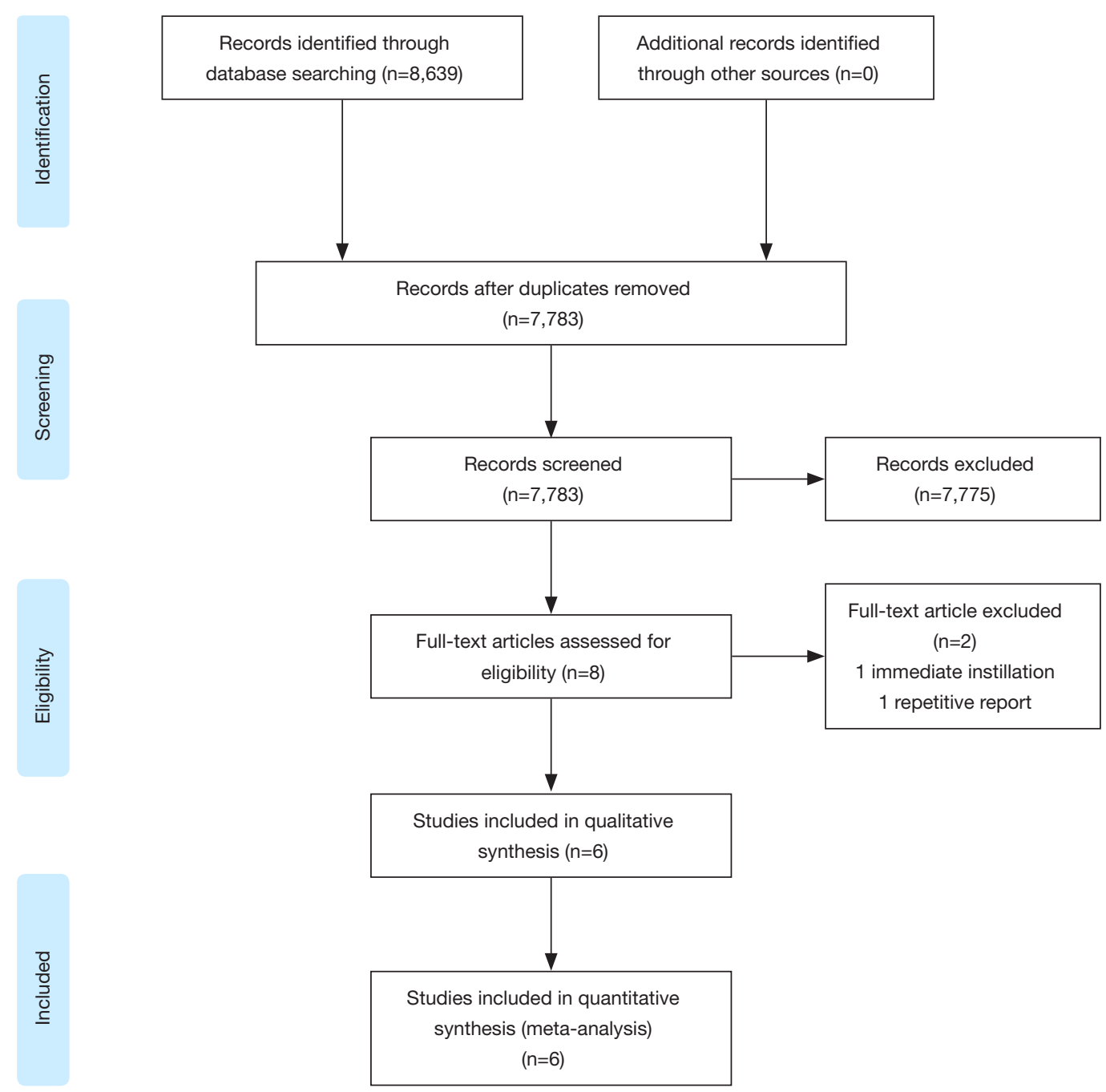

Figure 1 Study selection flowchart.

for both RFS and PFS. All the pooled effect sizes after omitting study are in the $95 \%$ CI range of overall effect size, reflecting the robustness of the results.

\section{Discussion}

In this study, we carried out a systematic review and metaanalysis to evaluate the efficacy and toxicity of GEM and BCG in patients with NMIBC. The results demonstrated that GEM and BCG had similar effects in preventing tumor recurrence and progression. In a subgroup of patients with high risk of recurrence, the result did not display an advantage in any of the drugs. However, compared with BCG, patients treated with GEM were less likely to develop dysuria and hematuria. Although the number of adverse events was equal between the two drugs, patients treated with BCG seemed to be more likely to have multiple adverse events at the same time. These results showed that GEM had a favorable efficacy and less toxicity, revealing that it could be used as an alternative to BCG.

Gemcitabine, as a systematic chemotherapeutic agent, has been used to treat many types of tumor, such as bladder cancer, pancreatic adenocarcinoma, non-small cell lung cancer, and breast cancer (22-24). In bladder cancer, GEM combined with a platinum chemotherapeutic drug is the standard treatment of MIBC, which indicating its efficacy in intravenous application (1). In recent years, GEM has also been used to treat NMIBC through intravesical instillation, including immediate instillation and maintenance instillation. Clinical trials of these two instillation strategies 


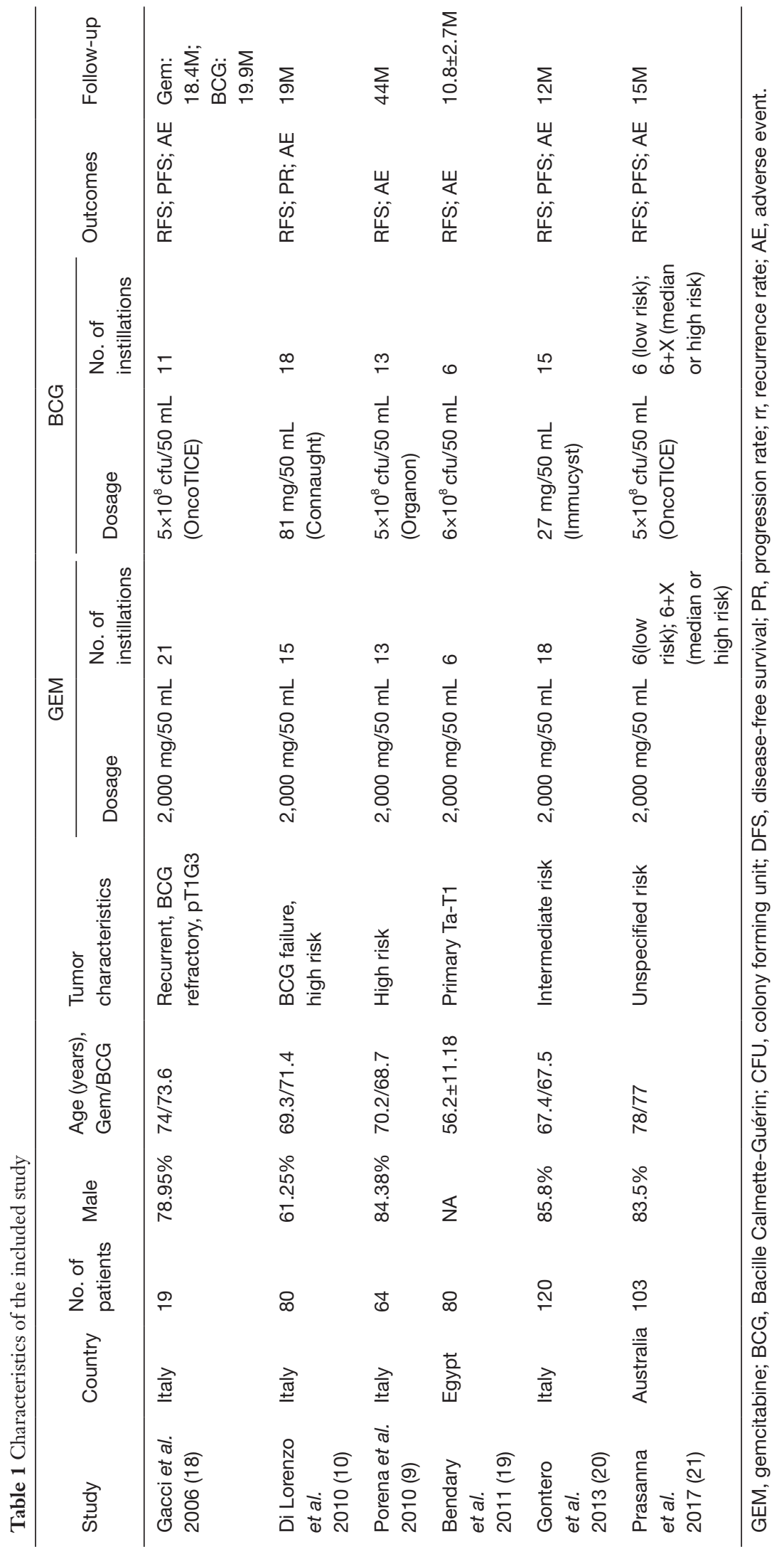




\begin{tabular}{|c|c|c|c|c|c|}
\hline Study & Hazard ratio & $95 \% \mathrm{CI}$ & Weight & & \\
\hline 1. Recurrence free survival & & & & Favor GEM & Favor BCG \\
\hline Gacci2006 & 0.62 & {$[0.22,1.76]$} & $12.63 \%$ & $\mapsto$ & $\longrightarrow$ \\
\hline Di Lorenzo2010 & 0.42 & {$[0.25,0.69]$} & $19.92 \%$ & -1 & \\
\hline Porena2010 & 2.08 & {$[1.04,4.19]$} & $17.25 \%$ & & $\longmapsto$ \\
\hline Bendary2011 & 0.83 & {$[0.36,1.92]$} & $15.31 \%$ & $\mapsto$ & $\longrightarrow$ \\
\hline Gontero2013 & 1.22 & {$[0.64,2.30]$} & $18.06 \%$ & $\mapsto$ & $=$ \\
\hline Prasanna2017 & 0.47 & {$[0.23,0.98]$} & $16.85 \%$ & 1 & \\
\hline Overall effect & 0.80 & {$[0.46,1.37]$} & $100 \%$ & 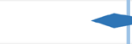 & r \\
\hline \multicolumn{6}{|c|}{ Heterogeneity: $\mathrm{Q}=17.25, \mathrm{P}=0.004 ; \mathrm{I} 2=71.0 \%$; } \\
\hline \multicolumn{6}{|c|}{ 2. Progression free survival } \\
\hline Gacci2006 & 0.45 & {$[0.08,25.02]$} & $0.18 \%$ & & $\rightarrow$ \\
\hline Di Lorenzo2010 & 0.55 & {$[0.23,1.32]$} & $93.10 \%$ & & $\longrightarrow$ \\
\hline Gontero2013 & 2.18 & {$[0.63,7.52]$} & $2.33 \%$ & & $\rightarrow$ \\
\hline Prasanna2017 & 0.53 & {$[0.06,5.08]$} & $4.39 \%$ & $\longmapsto$ & $\rightarrow$ \\
\hline pooled ES & 0.82 & {$[0.38,1.77]$} & $100 \%$ & 4 & \\
\hline Heterogeneity: $\mathrm{Q}=3.5, \mathrm{P}=$ & $\mathrm{I} 2=14.3 \%$ & & & 0 & 4 \\
\hline
\end{tabular}

Figure 2 Forest plot for recurrence free survival and progression free survival.

\begin{tabular}{|c|c|c|c|c|c|c|c|c|c|c|c|}
\hline \multirow{2}{*}{ Study } & \multicolumn{2}{|c|}{ GEM } & \multicolumn{2}{|c|}{ BCG } & \multirow{2}{*}{ Odds ratio } & \multirow{2}{*}{$95 \% \mathrm{CI}$} & \multirow{2}{*}{ Weight } & \multirow[b]{3}{*}{ Favor GEM } & \multirow[b]{3}{*}{ Favor BCG } & \\
\hline & Events & Total & Events & Total & & & & & & & \\
\hline \multicolumn{8}{|l|}{ 1. Total adverse events } & & & & \\
\hline Gacci2006 & 2 & 9 & 4 & 19 & 1.05 & {$[0.23,4.87]$} & $11.9 \%$ & & 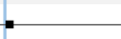 & 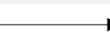 & - \\
\hline Di Lorenzo2010 & 15 & 40 & 16 & 40 & 0.96 & {$[0.53,1.74]$} & $33.4 \%$ & & 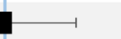 & & \\
\hline Gontero2013 & 14 & 41 & 19 & 47 & 0.88 & {$[0.49,1.60]$} & $33.7 \%$ & & & & \\
\hline Prasanna2017 & 4 & 51 & 23 & 52 & 0.24 & {$[0.09,0.65]$} & $21.1 \%$ & 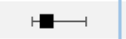 & & & \\
\hline Overall effect & & & & & 0.70 & {$[0.38,1.29]$} & $100.0 \%$ & & - & & \\
\hline \multicolumn{12}{|c|}{ Heterogeneity: $Q=6.50, P=0.090 ; \mathrm{I} 2=53.8 \%$; } \\
\hline \multicolumn{12}{|c|}{ 2. Dysuria } \\
\hline Gacci2006 & 0 & 9 & 1 & 10 & 0.40 & {$[0.02,8.78]$} & $3.3 \%$ & & & & $\rightarrow$ \\
\hline Di Lorenzo2010 & 6 & 40 & 8 & 40 & 0.78 & {$[0.29,2.08]$} & $32.5 \%$ & & $\longrightarrow$ & & \\
\hline Bendary2011 & 5 & 40 & 14 & 40 & 0.43 & {$[0.17,1.10]$} & $35.1 \%$ & & & & \\
\hline Gontero2013 & 4 & 41 & 15 & 47 & 0.37 & {$[0.13,1.03]$} & $29.1 \%$ & & & & \\
\hline Overall effect & & & & & 0.50 & {$[0.29,0.87]$} & $100.0 \%$ & $\sim$ & & & \\
\hline \multicolumn{12}{|c|}{ Heterogeneity: $\mathrm{Q}=1.28, \mathrm{P}=0.735 ; \mathrm{I} 2=0 \%$; } \\
\hline \multicolumn{12}{|c|}{ 3. Hematuria } \\
\hline Gacci2006 & 0 & 9 & 1 & 10 & 0.40 & {$[0.02,8.78]$} & $7.1 \%$ & $\longmapsto$ & & & $\rightarrow$ \\
\hline Di Lorenzo2010 & 2 & 40 & 5 & 40 & 0.43 & {$[0.09,2.09]$} & $26.8 \%$ & $\mapsto$ & $\longrightarrow$ & & \\
\hline Gontero2013 & 4 & 41 & 11 & 47 & 0.47 & {$[0.16,1.38]$} & $58.0 \%$ & 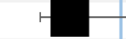 & $\longrightarrow$ & & \\
\hline Prasanna2017 & 0 & 51 & 5 & 52 & 0.10 & {$[0.01,1.79]$} & $8.2 \%$ & - & $\longrightarrow$ & & \\
\hline Overall effect & & & & & 0.40 & {$[0.18,0.91]$} & $100.0 \%$ & $\simeq$ & & & \\
\hline \multicolumn{12}{|c|}{ Heterogeneity: $\mathrm{Q}=1.02, \mathrm{P}=0.797 ; \mathrm{I} 2=0 \%$; } \\
\hline \multicolumn{12}{|c|}{ 4. Fever } \\
\hline Gacci2006 & 1 & 9 & 2 & 10 & 0.60 & {$[0.06,5.69]$} & $22.5 \%$ & $\longmapsto$ & & & $\rightarrow$ \\
\hline Porena2010 & 0 & 32 & 2 & 32 & 0.21 & {$[0.01,4.26]$} & $12.6 \%$ & $\vdash$ & & & $\rightarrow$ \\
\hline Di Lorenzo2010 & 1 & 40 & 3 & 40 & 0.35 & {$[0.04,3.23]$} & $23.0 \%$ & $\mapsto$ & & $\longrightarrow$ & \\
\hline Gontero2013 & 2 & 41 & 4 & 47 & 0.59 & {$[0.11,3.08]$} & $41.9 \%$ & $\longmapsto$ & & $\longrightarrow$ & \\
\hline Overall effect & & & & & 0.46 & {$[0.16,1.34]$} & $100.0 \%$ & & & & \\
\hline Heterogeneity: $\mathrm{Q}=0.47$, & $\mathrm{P}=0.926$ & $; 12=0$ & & & & & & 0 & 2 & 3 & 4 \\
\hline
\end{tabular}

Figure 3 Forest plot for adverse events. 
Table 2 Subgroup analysis for recurrence free survival (GEM vs. BCG)

\begin{tabular}{|c|c|c|c|c|c|c|}
\hline Subgroup & No. of study & Hazard ratio & $95 \% \mathrm{Cl}$ & Heterogeneity Q & $P_{h}$ & $\mathrm{I}^{2}$ \\
\hline \multicolumn{7}{|l|}{ Study design } \\
\hline $\mathrm{RCT}$ & 4 & 0.95 & {$[0.46,1.98]$} & 14.98 & 0.002 & $80.0 \%$ \\
\hline $\begin{array}{l}\text { Non-randomized } \\
\text { prospective study }\end{array}$ & 1 & 0.62 & {$[0.22,1.76]$} & - & - & - \\
\hline Europe & 4 & 0.90 & {$[0.41,1.97]$} & 15.30 & 0.002 & $80.4 \%$ \\
\hline Africa & 1 & 0.83 & {$[0.36,1.92]$} & - & - & - \\
\hline Oceania & 1 & 0.47 & {$[0.23,0.97]$} & - & - & - \\
\hline \multicolumn{7}{|l|}{ Study center } \\
\hline \multicolumn{7}{|l|}{ Schedule } \\
\hline Maintenance & 4 & 0.90 & {$[0.41,1.97]$} & 15.30 & 0.002 & $80.4 \%$ \\
\hline Induction & 1 & 0.83 & {$[0.36,1.92]$} & - & - & - \\
\hline \multicolumn{7}{|l|}{ Risk of recurrence } \\
\hline High risk & 3 & 0.81 & {$[0.28,2.39]$} & 13.32 & 0.001 & $85.0 \%$ \\
\hline Intermediate risk & 1 & 1.22 & {$[0.64,2.31]$} & - & - & - \\
\hline Unspecified risk & 2 & 0.60 & {$[0.35,1.04]$} & 1.01 & 0.314 & $1.30 \%$ \\
\hline
\end{tabular}

$\mathrm{RCT}$, randomized controlled trial; CCS, case-control study; $\mathrm{P}_{\mathrm{h}}, \mathrm{P}$ for heterogeneity.

demonstrated that GEM could prevent bladder tumor recurrence effectively $(10,25)$. Especially in patients with high risk or BCG failure NMIBC, the effect of GEM was promising.

In the meta-analysis, two studies recruit BCG failure patients. Di Lorenzo et al. found that $52.5 \%$ of patients developed disease recurrence after GEM treatment, whereas $87.5 \%$ of patients relapsed after BCG treatment (10). Another pilot study includes a total of 19 participants with pT1G3 tumor (18). Three in nine patients are recurrence-free after GEM instillation (18). However, in BCG group, only one patient is disease-free, and two patients die (18). A retrospective study with 69 patients analyzed the effect of GEM in different BCG failure types (BCG refractory, BCG resistant, BCG recurrent, and BCG intolerant) (26). The overall recurrence rate is reported as $66.7 \%$, and no difference is detected between BCG refractory and other BCG failure types (26). These results provide a feasible conservative treatment for patients with
BCG failure independent of failure type. In the metaanalysis, we perform a subgroup analysis by different risks of recurrence. All subgroups (high risk, intermediate-risk, and unspecified risk) show comparable efficacies between GEM and BCG. In unspecified risk subgroup, GEM treatment is more effective than BCG treatment but this is not statistically significant $[\mathrm{HR}=0.60(0.35-1.04)]$. Further studies of GEM treatment for non-high-risk tumor are required.

The total incidence of adverse events is similar between BCG and GEM groups. Patients treated with BCG are more likely to suffer urinary adverse events, such as dysuria, hematuria, cystitis, and even urosepsis $(20,21)$. This may be due to the strong local immune response of urothelial cells to live-attenuated Mycobacterium Bovis. Nevertheless, GEM is more likely to cause gastrointestinal tract and skin reactions, such as nausea and dermatitis, even though the incidence is rather low $(10,20)$. Gontero et al. measured the quality of life of patients receiving GEM and BCG (20). 
A Recurrence free survival

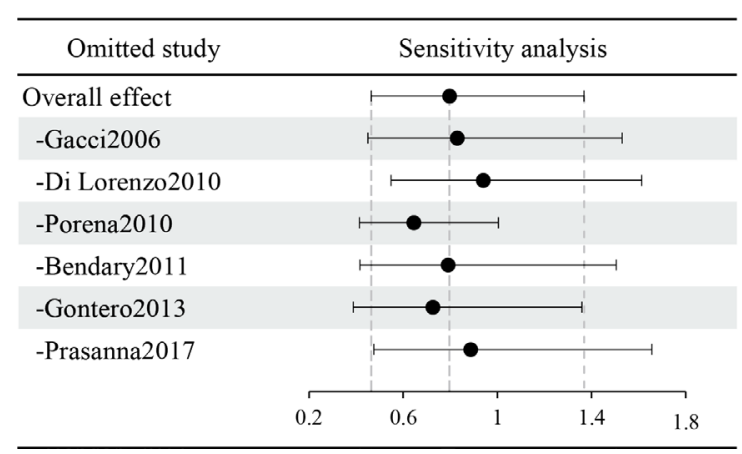

\section{B Progression free survival}

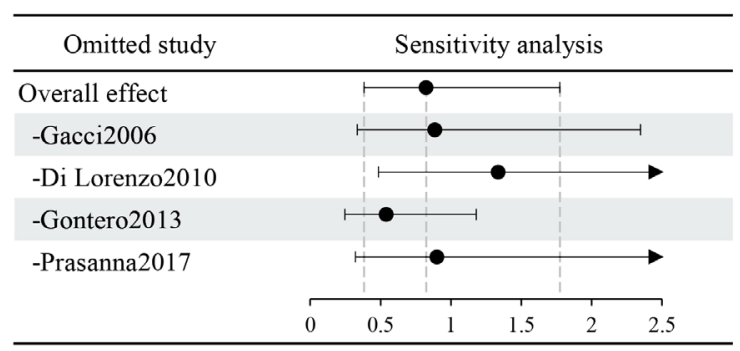

Figure 4 Sensitivity analysis for (A) recurrence free survival and (B) progression free survival.

The cognitive functioning score is significantly decreased in BCG group than GEM group over time (20). These results conclude that GEM treatment is associated with less toxicity than BCG treatment during regular instillation.

This study combines 6 original studies and provided an alternative intravesical therapy for BCG. The subgroup and sensitivity analysis reveal the robustness of the results. But there are still several limitations. The main limitation is the quality of the included studies. Three studies are of high risk of bias, and two studies are not randomized trials. Considering that different study types are included, we perform subgroup analysis and indicate similar effect sizes between different study designs. Another limitation is the small number of included studies and participants. It disenables the performance of publication bias tests and meta-regression to detect confounding factors. Further well design double-blind RCTs are required to assess the efficacy of scheduled GEM intravesical therapy in patients with NMIBC.

\section{Conclusions}

Scheduled intravesical GEM instillation has a similar effect with BCG instillation in preventing NMIBC recurrence and progression, but GEM therapy causes a lower incidence of dysuria and hematuria than BCG. GEM may be an alternative therapy for BCG. However, the results should be treated with caution due to the low to moderate quality of the included studies.

\section{Acknowledgments}

Here we thank Prof. Hu Jia for his professional guidance to polish our manuscript.
Funding: None.

\section{Footnote}

Reporting Checklist: The authors have completed the PRISMA reporting checklist. Available at https://dx.doi. org/10.21037/tcr-21-291

Conflicts of Interest: All authors have completed the ICMJE uniform disclosure form (available at https://dx.doi. org/10.21037/tcr-21-291). The authors have no conflicts of interest to declare.

Ethical Statement: The authors are accountable for all aspects of the work in ensuring that questions related to the accuracy or integrity of any part of the work are appropriately investigated and resolved.

Open Access Statement: This is an Open Access article distributed in accordance with the Creative Commons Attribution-NonCommercial-NoDerivs 4.0 International License (CC BY-NC-ND 4.0), which permits the noncommercial replication and distribution of the article with the strict proviso that no changes or edits are made and the original work is properly cited (including links to both the formal publication through the relevant DOI and the license). See: https://creativecommons.org/licenses/by-nc-nd/4.0/.

\section{References}

1. Babjuk M, Burger M, Compérat E, et al. EAU Guidelines. Edn. presented at the EAU Annual Congress Amsterdam 2020. ISBN 978-94-92671-07-3. 2020;EAU Guidel.

2. Arends TJ, Nativ O, Maffezzini M, et al. Results of a 
Randomised Controlled Trial Comparing Intravesical Chemohyperthermia with Mitomycin C Versus Bacillus Calmette-Guérin for Adjuvant Treatment of Patients with Intermediate- and High-risk Non-Muscle-invasive Bladder Cancer. Eur Urol 2016;69:1046-52.

3. Herr HW, Schwalb DM, Zhang ZF, et al. Intravesical bacillus Calmette-Guérin therapy prevents tumor progression and death from superficial bladder cancer: tenyear follow-up of a prospective randomized trial. J Clin Oncol 1995;13:1404-8.

4. Canyilmaz E, Yoney A, Serdar L, et al. Long-term results of a concomitant boost radiotherapy technique for elderly patients with muscle-1nvasive bladder cancer. J Geriatr Oncol 2015;6:316-23.

5. Giacalone NJ, Shipley WU, Clayman RH, et al. Longterm Outcomes After Bladder-preserving Tri-modality Therapy for Patients with Muscle-invasive Bladder Cancer: An Updated Analysis of the Massachusetts General Hospital Experience. Eur Urol 2017;71:952-60.

6. Mak KS, Smith AB, Eidelman A, et al. Quality of Life in Long-term Survivors of Muscle-Invasive Bladder Cancer. Int J Radiat Oncol Biol Phys 2016;96:1028-36.

7. Chen S, Zhang N, Shao J, et al. Maintenance versus non-maintenance intravesical Bacillus Calmette-Guerin instillation for non-muscle invasive bladder cancer: A systematic review and meta-analysis of randomized clinical trials. Int J Surg 2018;52:248-57.

8. Mostafid AH, Palou Redorta J, Sylvester R, et al. Therapeutic options in high-risk non-muscle-invasive bladder cancer during the current worldwide shortage of bacille Calmette-Guérin. Eur Urol 2015;67:359-60.

9. Porena M, Del Zingaro M, Lazzeri M, et al. Bacillus Calmette-Guerin versus gemcitabine for intravesical therapy in high-risk superficial bladder cancer: a randomised prospective study. Urol Int 2010;84:23-7.

10. Di Lorenzo G, Perdonà S, Damiano R, et al. Gemcitabine versus bacille Calmette-Guérin after initial bacille Calmette-Guérin failure in non-muscle-invasive bladder cancer: a multicenter prospective randomized trial. Cancer 2010;116:1893-900.

11. Ye Z, Chen J, Hong Y, et al. The efficacy and safety of intravesical gemcitabine vs bacille calmette-guerin for adjuvant treatment of non-muscle invasive bladder cancer: A meta-analysis. Onco Targets Ther 2018;11:4641-9.

12. Page MJ, Moher D, Bossuyt PM, et al. PRISMA 2020 explanation and elaboration: updated guidance and exemplars for reporting systematic reviews. BMJ 2021;372:n160.
13. Parmar MK, Torri V, Stewart L. Extracting summary statistics to perform meta-analyses of the published literature for survival endpoints. Stat Med 1998;17:2815-34.

14. Tierney JF, Stewart LA, Ghersi D, et al. Practical methods for incorporating summary time-to-event data into metaanalysis. Trials 2007;8:16.

15. Sterne JAC, Savović J, Page MJ, et al. RoB 2: a revised tool for assessing risk of bias in randomised trials. BMJ 2019;366:14898.

16. Sterne JA, Hernán MA, Reeves BC, et al. ROBINS-I: a tool for assessing risk of bias in non-randomised studies of interventions. BMJ 2016;355:i4919.

17. The Newcastle-Ottawa Scale (NOS) for assessing the quality of non randomised studies in meta-analyses. Available online: http://www.ohri.ca/programs/clinical_ epidemiology/oxford.asp

18. Gacci M, Bartoletti R, Cai T, et al. Intravesical gemcitabine in BCG-refractory T1G3 transitional cell carcinoma of the bladder: A pilot study. Urol Int 2006;76:106-11.

19. Bendary L, Khalil S, Shahin A, et al. Intravesical Gemcitabine versus bacillus calmette-guerin (BCG) in treatment of non-muscle invasive bladder cancer: Short term comparative study. J Urol 2011;1:e664-5.

20. Gontero P, Oderda M, Mehnert A, et al. The impact of intravesical gemcitabine and 1/3 dose Bacillus CalmetteGuérin instillation therapy on the quality of life in patients with nonmuscle invasive bladder cancer: results of a prospective, randomized, phase II trial. J Urol 2013;190:857-62.

21. Prasanna T, Craft P, Balasingam G, et al. Intravesical Gemcitabine versus Intravesical Bacillus Calmette-Guérin for the Treatment of Non-Muscle Invasive Bladder Cancer: An Evaluation of Efficacy and Toxicity. Front Oncol 2017;7:260.

22. Binenbaum Y, Na'ara S, Gil Z. Gemcitabine resistance in pancreatic ductal adenocarcinoma. Drug Resist Updat 2015;23:55-68.

23. Zhong WZ, Chen KN, Chen C, et al. Erlotinib Versus Gemcitabine Plus Cisplatin as Neoadjuvant Treatment of Stage IIIA-N2 EGFR-Mutant Non-Small-Cell Lung Cancer (EMERGING-CTONG 1103): A Randomized Phase II Study. J Clin Oncol 2019;37:2235-45.

24. Iyengar NM, Smyth LM, Lake D, et al. Efficacy and Safety of Gemcitabine With Trastuzumab and Pertuzumab After Prior Pertuzumab-Based Therapy Among Patients With Human Epidermal Growth Factor Receptor 2-Positive Metastatic Breast Cancer: A Phase 2 Clinical Trial. JAMA 
Netw Open 2019;2:e1916211.

25. Messing EM, Tangen CM, Lerner SP, et al. Effect of Intravesical Instillation of Gemcitabine vs Saline Immediately Following Resection of Suspected LowGrade Non-Muscle-Invasive Bladder Cancer on Tumor Recurrence: SWOG S0337 Randomized Clinical Trial.

Cite this article as: Lu JL, Xia QD, Liu CQ, Sun JX, Yang YY, Hu HL, Wang SG. Efficacy and toxicity in scheduled intravesical gemcitabine versus Bacille Calmette-Guérin for Ta and T1 bladder cancer: a systematic review and metaanalysis. Transl Cancer Res 2021;10(6):2849-2858. doi: 10.21037/tcr-21-291
JAMA 2018;319:1880-8.

26. Sternberg IA, Dalbagni G, Chen LY, et al. Intravesical Gemcitabine for High Risk, Nonmuscle Invasive Bladder Cancer after Bacillus Calmette-Guerin Treatment Failure. J Urol 2013;190:1686-91. 


\section{Supplementary}

Table S1 Quality assessment using the Newcastle-Ottawa Scale for case-control study

\begin{tabular}{|c|c|c|c|c|c|c|c|c|c|}
\hline \multirow[b]{2}{*}{ Study } & \multicolumn{4}{|c|}{ Selection } & \multicolumn{2}{|l|}{ Comparability } & \multicolumn{2}{|l|}{ Outcomes } & \multirow[b]{2}{*}{ Scores } \\
\hline & $\begin{array}{l}\text { Representativeness } \\
\text { of the exposed } \\
\text { cohort }\end{array}$ & $\begin{array}{l}\text { Selection of the } \\
\text { non-exposed } \\
\text { cohort }\end{array}$ & $\begin{array}{l}\text { Ascertainment } \\
\text { of exposure }\end{array}$ & $\begin{array}{l}\text { Demonstration that } \\
\text { outcome of interest } \\
\text { was not present at } \\
\text { start of study }\end{array}$ & $\begin{array}{l}\text { Comparability of } \\
\text { cohorts on the } \\
\text { basis of the design } \\
\text { or analysis }\end{array}$ & $\begin{array}{l}\text { Assessment } \\
\text { of outcome }\end{array}$ & $\begin{array}{l}\text { Was follow-up } \\
\text { long enough for } \\
\text { outcomes to } \\
\text { occur }\end{array}$ & $\begin{array}{l}\text { Adequacy } \\
\text { of follow up } \\
\text { of cohorts }\end{array}$ & \\
\hline $\begin{array}{l}\text { Prasanna, } \\
\text { et al., } \\
2017\end{array}$ & is & ț & is & 糸 & 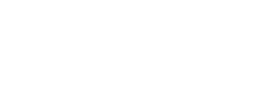 & 弥 & $\xi$ & 㶦 & 8 谉 \\
\hline
\end{tabular}

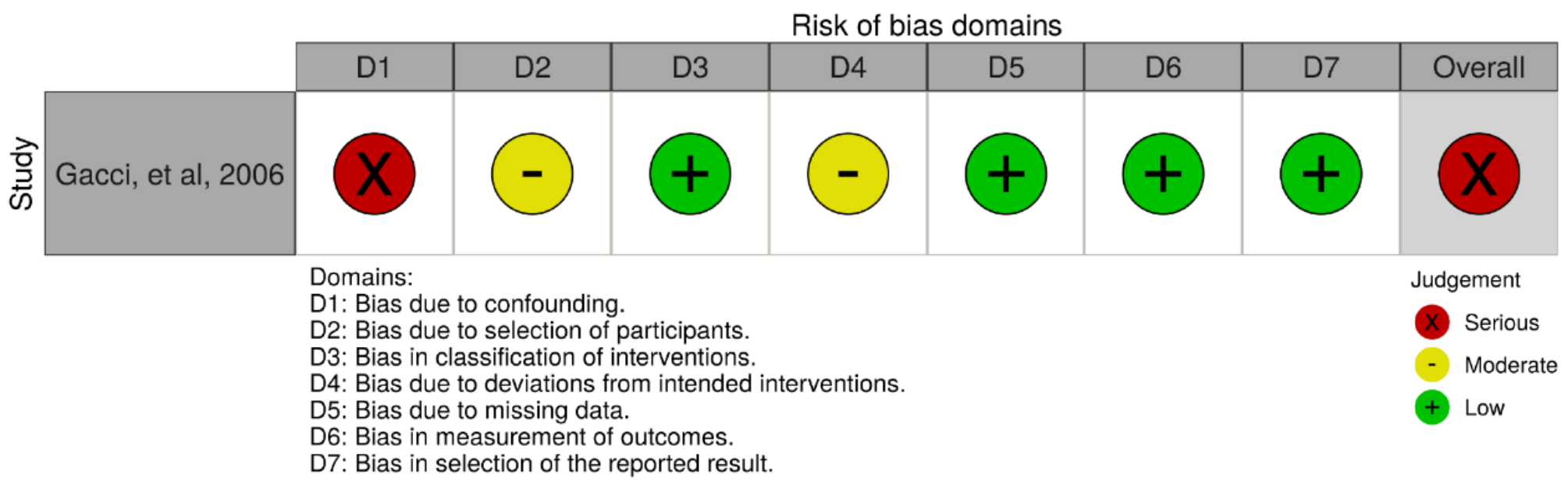

Figure S1 Quality assessment using the Risk Of Bias In Non-randomized Studies of Interventions for prospective intervention study. 


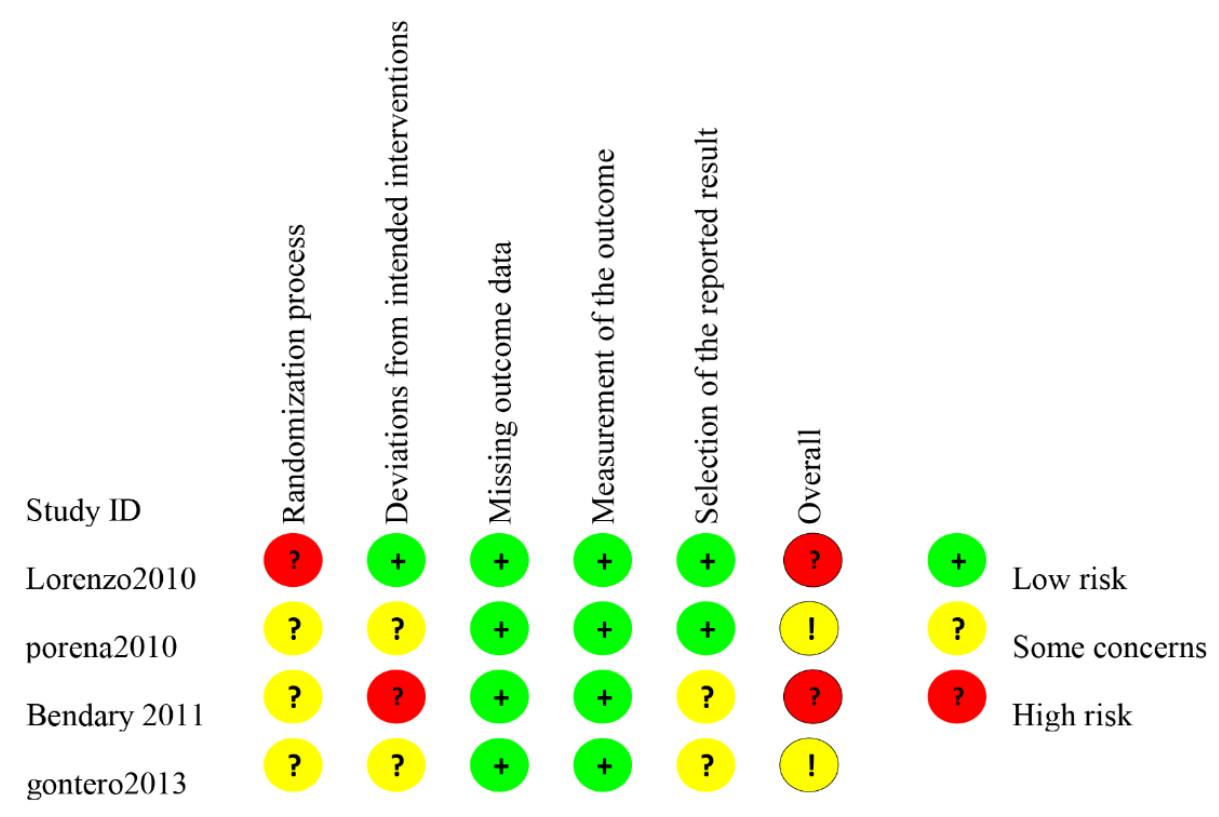

Figure S2 Quality assessment using RoB 2 for randomized controlled study.

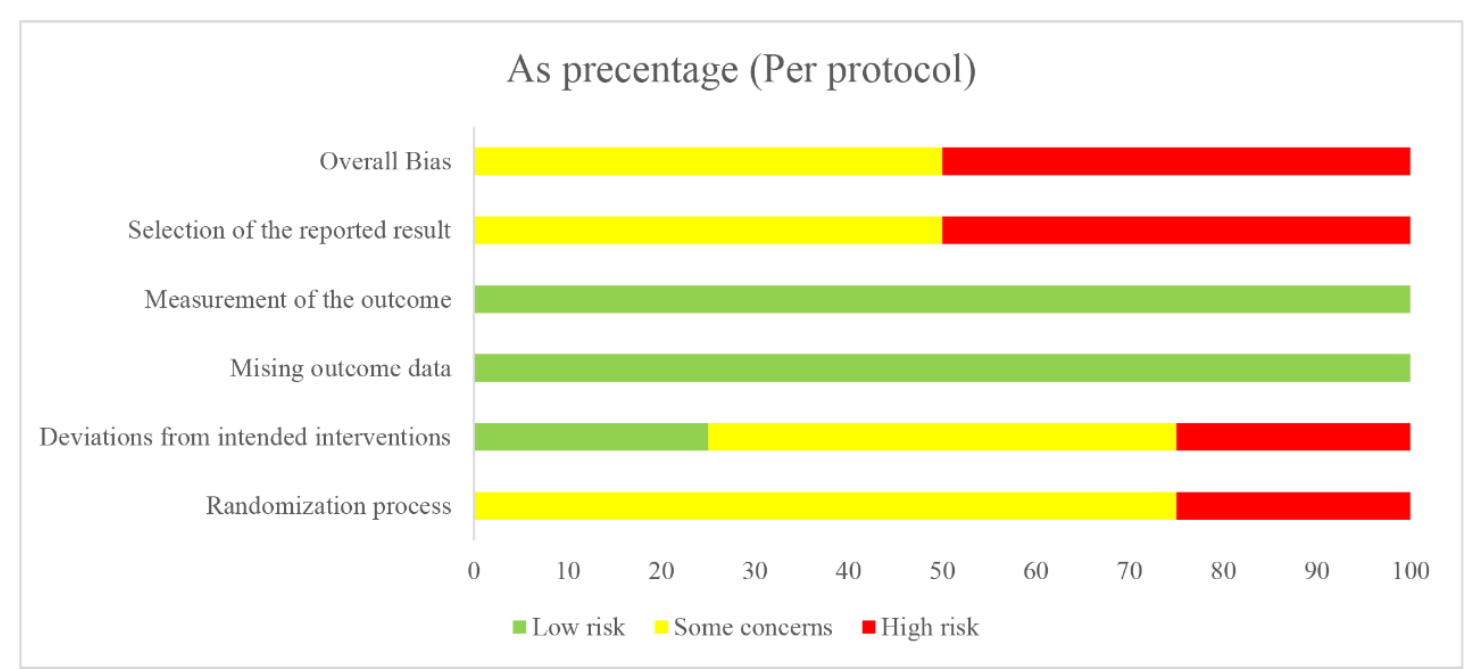

Figure S3 Quality assessment summary using RoB 2 for randomized controlled study. 